Research Article

\title{
Similarity Requirements for Mixed Convective Boundary Layer Flow over Vertical Curvilinear Porous Surfaces with Heat Generation/Absorption
}

\author{
Kh. Abdul Maleque \\ American International University-Bangladesh, Kuratali, Khilkhat, Dhaka 1219, Bangladesh \\ Correspondence should be addressed to Kh. Abdul Maleque; maleque@aiub.edu
}

Received 27 July 2019; Revised 5 February 2020; Accepted 17 February 2020; Published 3 March 2020

Academic Editor: Andre Cavalieri

Copyright (C) $2020 \mathrm{Kh}$. Abdul Maleque. This is an open access article distributed under the Creative Commons Attribution License, which permits unrestricted use, distribution, and reproduction in any medium, provided the original work is properly cited.

\begin{abstract}
Similarity requirements for three dimensional combined forced and free convective laminar boundary layer flows over the porous inclined vertical curvilinear surfaces with buoyancy effects and heat absorption/generation effects are investigated theoretically. The potential flow in the mainstream and Gabriel lame coefficients outside of the boundary layer are the function of $(\xi, \eta)$. Hence, the external velocity components $\left(U_{e}, V_{e}\right)$ and Gabriel lame coefficients $\left(h_{1}, h_{2}, h_{3}\right)$ are independent of $\zeta$. Here, $h_{3}(\xi, \eta)=1$ has been set such that $\zeta$ represents actual distance measured normal to the surface. Similarity requirements for an incompressible fluid are sought on the basis of detailed analyses in order to reduce the governing partial differential equations into a set of ordinary differential equations. Finally, different possible cases are exhibited in a tabular form with the inclusion of $\Delta T$ variations for onward flow study that are helpful to the future researchers for the flow over the orthogonal curvilinear surfaces.
\end{abstract}

\section{Introduction}

When a viscous fluid flows over a heated (or cooled) vertical orthogonal curved surface in inclined position, then velocity and thermal boundary layers are formed over the surface. The similarity equations exist only for a very special type of the component of main stream flow $\left(U_{e}(\xi, \eta), V_{e}(\xi, \eta), 0\right)$ and temperature difference $\Delta T(\xi, \eta)$ between the wall and ambient fluid. Howarth [1] investigated the equations of boundary layer flow in the vicinity of a separation point on a general three-dimensional case. He showed that the equations were reducible to a pair of simultaneous ordinary differential equations. Devy and Schofield [2] studied the same case and presented numerical results in a tabular form. Hansen $[3,4]$ presented a table showing the nature of the variations in the mainstream components for which the governing equations were reducible to a set of ordinary differential equations. Restrictions on main flow velocity components and coordinate system which leads to similarity solutions were given in a tabular form. Wang [5] discussed general methods for calculating three-dimensional laminar boundary layers over inclined blunt bodies (not necessarily bodies of revolution). He used the traditional integral method and presented complete incompressible boundary layer results for a prolate spheroid at $30^{\circ}$ incidence. Cheng [6] investigated the combined free and forced convection boundary layer flow along inclined surfaces embedded in porous media. It was found that similarity solutions exit when both the wall temperature distribution of the plate outside of the boundary layer vary according to some power function of distance. Langston et al. [7] studied the detailed measurements of the subsonic flow in a large scale; plane turbine cascade was made to evaluate the three-dimensional nature of the flow field. Raju et al. [8] considered the boundary layer flow over a semi-infinite vertical and horizontal flat plate, heated to a constant temperature in a uniform free stream. They discussed when the buoyancy forces either aid or oppose the development of the boundary layer. Different mixed convection parameters were introduced in the formation of the respective problems involving horizontal and vertical surfaces such that smooth transition from one convection limit to the other was possible; the governing equations for the purely forced and free convection cases were, respectively, recovered from the zero values of Grashof and Reynolds 

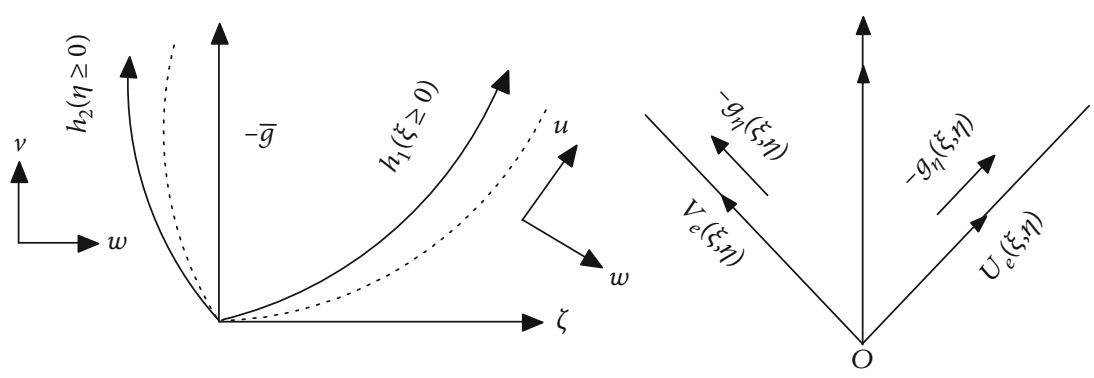

Figure 1: The flow configuration and the coordinate system.

numbers. A quite good number of theoretical studies of such a flow under free convection are found in literature. Merkin and Mahmood [9] introduced the logarithmic terms in their perturbation technique, the leading order solution being of $O\left[(\log )^{-1}\right]$. A general classification is made by the threedimensional boundary layer flow for both compressible and incompressible fluids by Chong and Perry [10]. Chen and Chang [11] presented an analysis to predict the heat transfer rate between two natural convection systems at different temperature separated by a vertical plate. They opined that their predicted results matched well with the reliable experimental data. The effects of temperature difference in order to predict the frictional resistance and heat transfer rate by introducing the components of the fluid velocity generated by buoyancy effects in the direction parallel to the edges of the surface were discussed by Zakerullah and Maleque [12]. In their another paper [13], they also investigated the nature of free stream velocities and temperature difference variations between the ambient fluid and the threedimensional rectangular surfaces theoretically and numerically. Numerical solution of MHD three-dimensional free convection and mass transfer flow with Hall current, viscous dissipation, and Joule heating was discussed by Sattar and Maleque [14]. The effects of magnetic field and heat absorption on three-dimensional transient convective boundary layer flow were studied by Maleque and Sattar [15]. They also studied the combined convective boundary layer flow due to a rotating disk with a Hall effect [16]. Steady threedimensional forced convective compressible fluid flow with variable properties and Hall current was discussed by Maleque and Sattar $[17,18]$. Effects of combined temperatureand depth-dependent viscosity and Hall current on unsteady fully three-dimensional magnetohydrodynamic boundary layer flow were studied by Maleque [19]. Receptivity of localized surface roughness and free-stream vorticity in threedimensional boundary layer flow was considered by Schrader et al. [20]. Maleque [21] investigated the effect of thermal diffusion on MHD convective heat and mass transfer flow due to a rotating disk. Steady hydrodynamic flow of a powerlaw non-Newtonian fluid from a rotating disk to a saturated non-Darcian porous medium was studied by Beg et al. [22]. Nadeem et al. [23] studied three-dimensional Casson fluid flow past a porous linearly stretching sheet. In their another paper [24], they also investigated the MHD effect with the fluid and convective condition along the three-dimensional surface. The three-dimensional boundary layer flow and heat transfer of a Cu-water on a permeable shrinking flat surface were studied by Mohd Noor and Roslinda [25]. MHD three-dimensional incompressible boundary layer flow that is generated by a bidirectional stretching surface was recently investigated by Hayat et al. [26]. Maleque [27, 28] investigated the effect of a uniform electric field and Hall current on unsteady MHD three-dimensional non-Newtonian Casson fluid flow due to a porous rotating disk. Recently, Hayat et al. [29] studied the effect of chemical reaction on threedimensional combined convective boundary layer flow of Williamson nanofluid. More recently, Rashid et al. [30] considered three-dimensional nanomaterial flow of Maxwell material over a stretchable moving rotating frame that was generated by a linear stretched sheet.

The present paper concerned with the systematic analyses in reducing the governing partial differential equations for a combined convective laminar incompressible flow over the inclined orthogonal curvilinear surfaces into a set of ordinary differential equations. Finally, a tabular form of similarity requirements is made for $\Delta T, h_{1}, h_{2}, U_{e}$, and $V_{e}$ variations. Two parameters $U_{F}^{2} / U_{e}^{2}$ and $V_{F}^{2} / V_{e}^{2}$ are introduced to show the dominancy of buoyancy effect over the forced flow effect, where $\left(U_{F}(\xi, \eta), V_{F}(\xi, \eta), 0\right)$ are the local fluid velocity components generated by buoyancy effect only and $\left(U_{e}(\xi, \eta), V_{e}(\xi, \eta), 0\right)$ are the components of the main stream velocity.

\section{Geometry of the Problem and the Governing Equations}

An orthogonal curvilinear heated surface inclined in its own position is held vertically in the free-stream velocity $\left(U_{e}(\xi\right.$, $\left.\eta), V_{e}(\xi, \eta), 0\right)$ perpendicular to $\zeta$ axis shown in Figure 1.

$\bar{g}\left(-g_{\xi}(\xi, \eta),-g_{\eta}(\xi, \eta), 0\right)$ is the acceleration vector of the fluid particle due to buoyancy effect of the fluid along $\xi$ and $\eta$ increasing owing to heated surface. With the introduction of Prandtl boundary layer concept, the equations governing the flow field in general orthogonal curvilinear coordinates are

$$
\begin{aligned}
& \frac{\partial}{\partial \xi}\left(h_{2} u\right)+\frac{\partial}{\partial \eta}\left(h_{1} v\right)+\frac{\partial}{\partial \zeta}\left(h_{1} h_{2} w\right)=0 \\
& \rho\left(\frac{u}{h_{1}} \frac{\partial u}{\partial \xi}+\frac{v}{h_{2}} \frac{\partial u}{\partial \eta}+w \frac{\partial u}{\partial \zeta}+\frac{u v}{h_{1} h_{2}} \frac{\partial h_{1}}{\partial \eta}-\frac{v^{2}}{h_{1} h_{2}} \frac{\partial h_{2}}{\partial \xi}\right) \\
& \quad=-\frac{1}{h_{1}} \frac{\partial p}{\partial \xi}+\rho g_{\xi}+\mu \frac{\partial^{2} u}{\partial \zeta^{2}}
\end{aligned}
$$




$$
\begin{aligned}
& \rho\left(\frac{u}{h_{1}} \frac{\partial v}{\partial \xi}+\frac{v}{h_{2}} \frac{\partial v}{\partial \eta}+w \frac{\partial v}{\partial \zeta}+\frac{u v}{h_{1} h_{2}} \frac{\partial h_{2}}{\partial \xi}-\frac{u^{2}}{h_{1} h_{2}} \frac{\partial h_{1}}{\partial \eta}\right) \\
& \quad=-\frac{1}{h_{2}} \frac{\partial p}{\partial \eta}+\rho g_{\eta}+\mu \frac{\partial^{2} v}{\partial \zeta^{2}}, \\
& \frac{\partial p}{\partial \zeta}=0,
\end{aligned}
$$$$
\rho\left(\frac{u}{h_{1}} \frac{\partial T}{\partial \xi}+\frac{v}{h_{2}} \frac{\partial T}{\partial \eta}+w \frac{\partial T}{\partial \zeta}\right)=\frac{\mu}{P_{r}} \frac{\partial^{2} T}{\partial \zeta^{2}}+\frac{Q_{0}}{C_{p}}\left(T-T_{e}\right) .
$$

Here, $h_{3}(\xi, \eta)=1$ has been set such that $\zeta$ represents actual distance measured normal to the surface. The body force terms are retained in equations (2) and (3) in addition to the equations used by Davy and Schofield [2]. The potential flow in the mainstream outside of the boundary layer is the function of $(\xi, \eta)$. Hence, the external velocity components $U_{e}$ and $V_{e}$ independent of $\zeta,(u, v, w)$ are the components of fluid velocity, and $T$ is the temperature in the boundary layer. $\mu$ and $\rho$ are the viscosity and the density of the fluid, respectively, $P_{r}$ is the Prandtl number, $C_{p}$ is the specific heat with constant pressure, and $Q_{0}$ is the heat generation $(>0)$ or absorption $(<0)$ coefficient.

The appropriate boundary conditions for the velocities and temperature fields are

$\left.\begin{array}{l}u=0, v=0, w=w_{0}(\xi, \eta), T=T_{w}(\xi, \eta), \text { at } \zeta=0, \\ u \longrightarrow U_{e}(\xi, \eta), v \longrightarrow V_{e}(\xi, \eta), T \longrightarrow T_{e} \text { as } \zeta \longrightarrow \infty .\end{array}\right\}$

$w_{0}$ is considered here to be the surface suction $\left(w_{0}>0\right)$ or injection $\left(w_{0}<0\right)$ velocity for the developable surface. $T_{w}$ and $T_{e}$ are the wall temperature and wall temperature, respectively.

To eliminate pressure terms in equations (2) and (3), the conditions outside the boundary layer are imposed. External conditions $u \longrightarrow U_{e}, v \longrightarrow V_{e}, \rho \longrightarrow \rho_{e}, T \longrightarrow T_{e}$ (=constant), $\partial / \partial \zeta \longrightarrow 0$, and the negligible pressure variation yield the following equations.

$$
\begin{aligned}
& \rho_{e}\left(\frac{U_{e}}{h_{1}} \frac{\partial U_{e}}{\partial \xi}+\frac{V_{e}}{h_{2}} \frac{\partial U_{e}}{\partial \eta}+\frac{U_{e} V_{e}}{h_{1} h_{2}} \frac{\partial h_{1}}{\partial \eta}-\frac{V_{e}^{2}}{h_{1} h_{2}} \frac{\partial h_{2}}{\partial \xi}\right)-\rho_{e} g_{\xi} \\
& =-\frac{1}{h_{1}} \frac{\partial p}{\partial \xi},
\end{aligned}
$$

$\rho_{e}\left(\frac{U_{e}}{h_{1}} \frac{\partial V_{e}}{\partial \xi}+\frac{V_{e}}{h_{2}} \frac{\partial V_{e}}{\partial \eta}+\frac{U_{e} V_{e}}{h_{1} h_{2}} \frac{\partial h_{2}}{\partial \xi}-\frac{U_{e}^{2}}{h_{1} h_{2}} \frac{\partial h_{1}}{\partial \eta}\right)-\rho_{e} g_{\eta}$

$$
=-\frac{1}{h_{2}} \frac{\partial p}{\partial \eta}
$$

$\rho_{e}\left(\frac{U_{e}}{h_{1}} \frac{\partial T_{e}}{\partial \xi}+\frac{V_{e}}{h_{2}} \frac{\partial T_{e}}{\partial \eta}\right)=0$.

$T_{e}=T_{0}$ (=constant) is also a solution of equation (9).

In view of equations (7) to (9), $T-T_{0}=\left(T_{w}-T_{0}\right) \theta$ $(\xi, \eta, \zeta)$ and $T_{w}-T_{0}=\Delta T$, the boundary layer and energy equations with the elimination of pressure terms are

$$
\begin{gathered}
\frac{\partial}{\partial \xi}\left(h_{2} u\right)+\frac{\partial}{\partial \eta}\left(h_{1} v\right)+\frac{\partial}{\partial \zeta}\left(h_{1} h_{2} w\right)=0, \\
\frac{u}{h_{1}} \frac{\partial u}{\partial \xi}+\frac{v}{h_{2}} \frac{\partial u}{\partial \eta}+w \frac{\partial u}{\partial \zeta}+\frac{u v}{h_{1} h_{2}} \frac{\partial h_{1}}{\partial \eta}-\frac{v^{2}}{h_{1} h_{2}} \frac{\partial h_{2}}{\partial \xi} \\
=-g_{\xi} \beta_{T} \Delta T \theta+v \frac{\partial^{2} u}{\partial \zeta^{2}}+\frac{U_{e}}{h_{1}} \frac{\partial U_{e}}{\partial \xi}+\frac{V_{e}}{h_{2}} \frac{\partial U_{e}}{\partial \eta} \\
+\frac{U_{e} V_{e}}{h_{1} h_{2}} \frac{\partial h_{1}}{\partial \eta}-\frac{V_{e}^{2}}{h_{1} h_{2}} \frac{\partial h_{2}}{\partial \xi},
\end{gathered}
$$

$$
\begin{aligned}
\frac{u}{h_{1}} \frac{\partial v}{\partial \xi} & +\frac{v}{h_{2}} \frac{\partial v}{\partial \eta}+w \frac{\partial v}{\partial \zeta}+\frac{u v}{h_{1} h_{2}} \frac{\partial h_{2}}{\partial \xi}-\frac{u^{2}}{h_{1} h_{2}} \frac{\partial h_{1}}{\partial \eta} \\
= & -g_{\eta} \beta_{T} \Delta T \theta+v \frac{\partial^{2} v}{\partial \zeta^{2}}+\frac{U_{e}}{h_{1}} \frac{\partial V_{e}}{\partial \xi}+\frac{V_{e}}{h_{2}} \frac{\partial V_{e}}{\partial \eta} \\
& +\frac{U_{e} V_{e}}{h_{1} h_{2}} \frac{\partial h_{2}}{\partial \xi}-\frac{U_{e}^{2}}{h_{1} h_{2}} \frac{\partial h_{1}}{\partial \eta},
\end{aligned}
$$

$$
\begin{aligned}
& \frac{u}{h_{1}} \frac{\partial \theta}{\partial \xi}+\frac{v}{h_{2}} \frac{\partial \theta}{\partial \eta}+w \frac{\partial \theta}{\partial \zeta}+\theta\left[\frac{u}{h_{1}}(\ln \Delta T)_{\xi}+\frac{v}{h_{2}}(\ln \Delta T)_{\eta}\right] \\
& \quad=\frac{v}{P_{r}} \frac{\partial^{2} \theta}{\partial \zeta^{2}}+\frac{Q_{0}}{\rho C_{p}} \theta .
\end{aligned}
$$

Considering $\rho=\rho(P, T)$. For Boussinesq fluid approximations, $\rho-\rho_{e}=-\rho \beta_{T} \Delta T \theta$ is substituted in the above equations where $\beta_{T}=-(1 / \rho)(\partial \rho / \partial T)_{p}$ is the coefficient of thermal expansion.

\section{Similarity Transformations}

Equations (10)-(13) are nonlinear, simultaneous partial differential equations, and the solutions of these equations are extremely difficult to obtain. Hence, our aim is to reduce equations (11)-(13) into ordinary differential equations with the help of equation (10). Let the set of variables $(\xi, \eta, \zeta)$ be changed to a new set of variables $(X, Y, \bar{\phi})$ where relations between two sets of variables are

$$
\begin{gathered}
X=\xi, \\
Y=\eta, \\
\zeta \overline{\gamma(X, Y)},
\end{gathered}
$$

$\gamma(X, Y)$ is considered here to be proportional to the square root of the local boundary layer thickness.

Let two stream functions $\psi(\xi, \eta, \zeta)$ and $\chi(\xi, \eta, \zeta)$ be defined as the mass flow components within the boundary layer for the case of incompressible flow. The following substitutions may be chosen 


$$
\begin{aligned}
\psi_{\zeta} & =h_{2} u, \\
\chi_{\zeta} & =h_{1} v, \\
-\left(\psi_{\xi}+\chi_{\eta}\right) & =h_{1} h_{2} w
\end{aligned}
$$

to satisfy the equation of continuity (10).

Guided by the idea of similarity, it can be allowed to write

$$
\begin{aligned}
& \int_{0}^{\bar{\phi}} \frac{u}{U(X, Y)} d \bar{\phi}=F(X, Y, \bar{\phi}), \\
& \int_{0}^{\bar{\phi}} \frac{v}{V(X, Y)} d \bar{\phi}=S(X, Y, \bar{\phi}) .
\end{aligned}
$$

In attempting separation of variables of $F(X, Y, \bar{\phi}), S(X$, $Y, \bar{\phi})$, and $\theta(X, Y, \bar{\phi})$, let it be assumed that

$$
\begin{aligned}
& F(X, Y, \bar{\phi})=L(X, Y) \bar{F}(\bar{\phi}), \\
& S(X, Y, \bar{\phi})=M(X, Y) \bar{S}(\bar{\phi}), \\
& \theta(X, Y, \bar{\phi})=N(X, Y) \bar{\theta}(\bar{\phi}),
\end{aligned}
$$

where $\bar{F}, \bar{S}$, and $\bar{\theta}$ are the functions of the single variable $\bar{\phi}$. Then, it can be found that

$$
\begin{aligned}
u= & U L \bar{F}_{\bar{\phi}}, \\
v= & V M \bar{S}_{\bar{\phi}}, \\
w= & -\frac{1}{h_{1} h_{2}}\left[\left(h_{2} \gamma U L\right)_{X} \bar{F}-\bar{\phi} \gamma_{X} h_{2} U L \bar{F}_{\bar{\phi}}\right. \\
& +\left(h_{1} \gamma V M\right)_{Y} \bar{S}-\bar{\phi} \gamma_{Y} h_{1} V M \bar{S}_{\bar{\phi}} \\
& \left.+h_{1} h_{2} w_{0}(X, Y, 0)\right] \\
\psi(X, Y, \bar{\phi})= & h_{2} \gamma U L \bar{F}(\bar{\phi})+\psi(X, Y, 0), \\
\chi(X, Y, \bar{\phi})= & h_{1} \gamma U L \bar{S}(\bar{\phi})+\chi(X, Y, 0),
\end{aligned}
$$

where $w_{0}(X, Y, 0)=-\left(1 / h_{1} h_{2}\right)\left[\psi_{X}(X, Y, 0)+\chi_{Y}(X, Y, 0)\right]$.

When the surface is porous, $w_{0}(X, Y, 0)$ represents the suction $\left(w_{0}>0\right)$ or injection $\left(w_{0}<0\right)$ velocity normal to the surface, since $U_{e}, V_{e}$ are independent of $\zeta$, then $\left(U_{e}\right)_{\bar{\phi}}=\left(V_{e}\right)_{\bar{\phi}}=0$. Equations (10), (11), (12), and (13) take the following forms:

$u$ - momentum equation:

$$
\begin{aligned}
& v \bar{F}_{\overline{\phi \phi}}+\frac{\gamma}{h_{1} h_{2}}\left(h_{2} \gamma U L\right)_{X} \bar{F} \bar{F}_{\overline{\phi \phi}}+\frac{\gamma}{h_{1} h_{2}}\left(h_{1} \gamma V M\right)_{Y} \bar{S} \bar{F}_{\overline{\phi \phi}} \\
& +\gamma w_{0} \bar{F}_{\overline{\phi \phi}}-\frac{\gamma^{2}}{h_{1}}(U L)_{X} \bar{F}_{\bar{\phi}}^{2}-\frac{\gamma^{2} V M}{h_{2}}\left[\frac{(U L)_{Y}}{U L}+\frac{h_{1 Y}}{h_{1}}\right] \bar{F}_{\bar{\phi}} \bar{S}_{\bar{\phi}} \\
& +\frac{\gamma^{2}(V M)^{2}}{h_{1} h_{2} U L} h_{2 X} \bar{S}_{\bar{\phi}}^{2}+\frac{\gamma^{2}}{h_{1} U L} U_{e} U_{e X}+\frac{\gamma^{2}}{h_{2} U L} V_{e} U_{e Y} \\
& +\frac{\gamma^{2} U_{e} V_{e}}{h_{1} h_{2} U L} h_{1 Y}-\frac{\gamma^{2} V_{e}^{2}}{h_{1} h_{2} U L} h_{2 X}-g_{X} \beta_{T} \frac{\gamma^{2}}{U L} \Delta T N \bar{\theta}=0 .
\end{aligned}
$$

$v$ - momentum equation:

$$
\begin{aligned}
& v \bar{S}_{\overline{\phi \phi \phi}}+\frac{\gamma}{h_{1} h_{2}}\left(\gamma h_{1} V M\right)_{Y} \bar{S}_{\overline{\phi \phi}}+\frac{\gamma}{h_{1} h_{2}}\left(\gamma h_{2} U L\right)_{X} \bar{F}_{\overline{\phi \phi}} \\
& +\gamma w_{0} \bar{S}_{\overline{\phi \phi}}-\frac{\gamma^{2}}{h_{2}}(V M)_{Y} \bar{S}_{\bar{\phi}}^{2}-\frac{\gamma^{2} U L}{h_{1}}\left[\frac{(V M)_{X}}{V M}+\frac{h_{2 X}}{h_{2}}\right] \bar{F}_{\bar{\phi}} \bar{S}_{\bar{\phi}} \\
& +\frac{\gamma^{2}(U L)^{2}}{h_{1} h_{2} V M} h_{1 Y} \bar{F}_{\bar{\phi}}^{2}+\frac{\gamma^{2}}{h_{2} V M} U_{e} V_{e X}+\frac{\gamma^{2}}{h_{1} V M} V_{e} V_{e Y} \\
& +\frac{\gamma^{2} U_{e} V_{e}}{h_{1} h_{2} V M} h_{2 X}-\frac{\gamma^{2} U_{e}^{2}}{h_{1} h_{2} V M} h_{1 Y}-g_{Y} \beta_{T} \frac{\gamma^{2}}{V M} \Delta T N \bar{\theta}=0 .
\end{aligned}
$$

Energy equation:

$$
\begin{aligned}
& \frac{v}{\operatorname{Pr}} \bar{\theta}_{\overline{\phi \phi}}+\frac{\gamma}{h_{1} h_{2}}\left(\gamma h_{2} U L\right)_{X} \bar{F} \bar{\theta}_{\bar{\phi}}+\frac{\gamma}{h_{1} h_{2}}\left(\gamma h_{1} V M\right)_{Y} \bar{S}_{\bar{\phi}} \\
& +\gamma w_{0} \bar{\theta}_{\bar{\phi}}+\gamma^{2} \frac{Q_{0} \Delta T}{\rho C_{p}} \bar{\theta}-\frac{\gamma^{2} U L}{h_{1}}\left[(\ln N)_{X}+(\ln \Delta T)_{X}\right] \bar{F}_{\bar{\phi}} \bar{\theta} \\
& \quad-\frac{\gamma^{2} V M}{h_{2}}\left[(\ln N)_{Y}+(\ln \Delta T)_{Y}\right] \bar{S}_{\bar{\phi}} \bar{\theta}=0 .
\end{aligned}
$$

In order to derive the generalized boundary conditions, we are to use the no slip conditions, exterior conditions for the velocity components, and finally the condition of the temperature function. Without loss of generality, we may put

$$
\begin{gathered}
U L=U_{e}, \\
V M=V_{e}, \\
N=1 .
\end{gathered}
$$

In view of (22), the equations (19)-(21) take the following form

$u$ - momentum equation:

$$
\begin{aligned}
& v \bar{F}_{\overline{\phi \phi \phi}}+\frac{1}{2}\left(a_{0}+a_{1}-a_{2}\right) \bar{F} \bar{F}_{\overline{\phi \phi}}+\frac{1}{2}\left(a_{3}+a_{4}-a_{5}\right) \bar{S} \bar{F}_{\overline{\phi \phi}} \\
& -a_{6} \bar{F}_{\overline{\phi \phi}}+a_{7}\left(1-\bar{F}_{\bar{\phi}}^{2}\right)+\left(a_{8}+a_{9}\right)\left(1-\bar{F}_{\bar{\phi}} \bar{S}_{\bar{\phi}}\right) \\
& -a_{10}\left(1-\bar{S}_{\bar{\phi}}^{2}\right)+a_{11} \bar{\theta}=0 .
\end{aligned}
$$

$v$ - momentum equation:

$$
\begin{aligned}
v \bar{S}_{\overline{\phi \phi \phi}}+\frac{1}{2}\left(a_{0}+a_{1}-a_{2}\right) \bar{F} \bar{S}_{\overline{\phi \phi}}+\frac{1}{2}\left(a_{3}+a_{4}-a_{5}\right) \bar{S}_{\overline{\phi \phi}} \\
-a_{6} \bar{S}_{\overline{\phi \phi}}+a_{12}\left(1-\bar{S}_{\bar{\phi}}^{2}\right)+\left(a_{13}+a_{14}\right)\left(1-\bar{F}_{\bar{\phi}} \bar{S}_{\bar{\phi}}\right) \\
-a_{15}\left(1-\bar{F}_{\bar{\phi}}^{2}\right)+a_{16} \bar{\theta}=0 .
\end{aligned}
$$

Energy equation:

$$
\begin{aligned}
& \frac{v}{\operatorname{Pr}} \bar{\theta}_{\overline{\phi \phi}}+\frac{1}{2}\left(a_{0}+a_{1}-a_{2}\right) \bar{F} \bar{\theta}_{\bar{\phi}}+\frac{1}{2}\left(a_{3}+a_{4}-a_{5}\right) \bar{S}_{\bar{\phi}} \\
& -a_{6} \bar{\theta}_{\bar{\phi}}-\left(a_{17} \bar{F}_{\bar{\phi}}+a_{18} \bar{S}_{\bar{\phi}}\right) \bar{\theta}+a_{19} \bar{\theta}=0,
\end{aligned}
$$


where $a_{0}, a_{1}, a_{2} \cdots a_{19}$ are the contractions in $X$ and $Y$ given by

$$
\begin{aligned}
& \text { (i) } a_{0}=\left(\frac{\gamma^{2} U_{e}}{h_{1}}\right)_{X} \text {, } \\
& \text { (ii) } a_{1}=\frac{\gamma^{2}\left(h_{2} U_{e}\right)_{X}}{h_{1} h_{2}} \text {, } \\
& \text { (iii) } a_{3}=\gamma^{2}\left(h_{2} U_{e}\right)\left(\frac{1}{h_{1} h_{2}}\right)_{X} \text {, } \\
& \text { (iv) } a_{3}=\left(\frac{\gamma^{2} V_{e}}{h_{2}}\right)_{Y} \text {, } \\
& (v) a_{4}=\frac{\gamma^{2}\left(h_{1} V_{e}\right)_{Y}}{h_{1} h_{2}} \text {, } \\
& \text { (vi) } a_{5}=\gamma^{2}\left(h_{1} V_{e}\right)\left(\frac{1}{h_{1} h_{2}}\right)_{Y} \text {, } \\
& \text { (vii) } a_{6}=-\gamma w_{0} \text {, } \\
& \text { (viii) } a_{7}=\frac{\gamma^{2} U_{e X}}{h_{1}} \text {, } \\
& \text { (ix) } a_{8}=\left(\frac{\gamma^{2} V_{e} U_{e Y}}{h_{2} U_{e}}\right) \text {, } \\
& (x) a_{9}=\gamma^{2} \frac{V_{e} h_{1 Y}}{h_{1} h_{2}}, \\
& \text { (xi) } a_{10}=\gamma^{2} \frac{V_{e}^{2} h_{2 X}}{h_{1} h_{2} U_{e}} \text {, } \\
& \text { (xii) } a_{11}=-\frac{\gamma^{2}}{U_{e}} g_{X} \beta_{T} \Delta T \text {, } \\
& \text { (xiii) } a_{12}=\gamma^{2} \frac{V_{e Y}}{h_{2}}, \\
& (x i v) a_{13}=\gamma^{2} \frac{U_{e} V_{e X}}{h_{1} V_{e}} \text {, } \\
& (x v) a_{14}=\gamma^{2} \frac{U_{e} h_{2 X}}{h_{1} h_{2}} \text {, } \\
& (x v i) a_{15}=\gamma^{2} \frac{U_{e}^{2} h_{1 Y}}{h_{1} h_{2} V_{e}} \text {, } \\
& \text { (xvii) } a_{16}=-\frac{\gamma^{2}}{V_{e}} g_{Y} \beta_{T} \Delta T \text {, } \\
& \left(\text { xviii) } a_{17}=\frac{\gamma^{2} U_{e}}{h_{1}}(\ln \Delta T)_{X}\right. \text {, } \\
& (x i x) a_{18}=\frac{\gamma^{2} V_{e}}{h_{2}}(\ln \Delta T)_{Y} \text {, } \\
& (x x) a_{19}=\gamma^{2} \frac{Q_{0} \Delta T}{\rho C_{p}} \text {. }
\end{aligned}
$$

Similarity solutions exist only when all the $a$ 's in equation (26) are finite and independent of $X$ and $Y$; that is to say that all $a$ 's must be constants.
Thus, the boundary layer momentum equations and energy equation will become nonlinear ordinary differential equations if $U_{e}(X, Y), V_{e}(X, Y), h_{1}(X, Y), h_{2}(X, Y), \gamma$ $(X, Y)$, and $\Delta T(X, Y)$ satisfy equation (26). From equations (26)(i) and (26)(iv) one may have

$$
\begin{aligned}
\frac{\gamma^{2} U_{e}}{h_{1}} & =a_{0} X+A(Y), \\
\frac{\gamma^{2} V_{e}}{h_{2}} & =a_{3} Y+B(X),
\end{aligned}
$$

where $A(Y)$ is the function of $Y$ alone and $B(X)$ is the function of $X$ alone. Using the equations in (26), $A(Y)$ and $B(X)$ are determined by the following pair of equations

$$
\begin{aligned}
& \frac{d A}{d Y}=\frac{h_{2} U_{e}}{h_{1} V_{e}}\left(a_{3}-a_{4}-a_{5}+a_{8}-a_{9}\right), \\
& \frac{d B}{d X}=\frac{h_{1} V_{e}}{h_{2} U_{e}}\left(a_{0}-a_{1}-a_{2}+a_{13}-a_{14}\right) .
\end{aligned}
$$

Equations (28) and (29) furnish us the possibilities of similarity cases for which we can find the nature of $U_{e}, V_{e}$, $h_{1}, h_{2}$, and finally $\Delta T$ in terms of $X$ and $Y$.

Case 1. Let $d A / d Y=$ constant and $d B / d X=$ constant.

This assumption yields

$$
\begin{aligned}
& \frac{V_{e}}{U_{e}}=k_{1}(=\text { constant }), \\
& \frac{h_{2}}{h_{1}}=k_{2}(=\text { constant }) .
\end{aligned}
$$

Let

$$
\begin{aligned}
& \frac{d A}{d Y}=\frac{k_{2}}{k_{1}} l_{1}, \\
& \frac{d B}{d X}=\frac{k_{1}}{k_{2}} l_{2},
\end{aligned}
$$

where $l_{1}=\left(a_{3}-a_{4}-a_{5}+a_{8}-a_{9}\right)$ and $l_{2}=\left(a_{0}-a_{1}-a_{2}+\right.$ $\left.a_{13}-a_{14}\right)$.

From equation (31), we have

$$
\begin{aligned}
& A(Y)=\frac{k_{2}}{k_{1}} l_{1} Y+A_{0}, \\
& B(X)=\frac{k_{1}}{k_{2}} l_{2} X+B_{0} .
\end{aligned}
$$

Then, equation (27) becomes

$$
\frac{\gamma^{2} U_{e}}{h_{1}}=a_{0} X+\frac{k_{2}}{k_{1}} l_{1} Y+A_{0},
$$




$$
\begin{aligned}
\frac{\gamma^{2} V_{e}}{h_{2}} & =a_{3} Y+\frac{k_{1}}{k_{2}} l_{2} X+B_{0}, \\
\text { or } \frac{\gamma^{2} U_{e}}{h_{1}} & =l_{2} X+\frac{k_{2}}{k_{1}} Y+B_{0}^{\prime} .
\end{aligned}
$$

Comparing equation (33) and equation (34), we have

$$
\begin{aligned}
& l_{2}=a_{0}, \\
& l_{1}=a_{3} \text { imply } a_{4}+a_{5}=a_{8}-a_{9} \text { and } a_{1}+a_{2}=a_{13}-a_{14},
\end{aligned}
$$

therefore

$$
\frac{\gamma^{2} U_{e}}{h_{1}}=a_{0} X+\frac{k_{2}}{k_{1}} a_{3} Y+A_{0} .
$$

In view of requirements (x) and (xi) of equation (26) and equation (36), one may derive

$$
h_{1}(x, y)=(a x+b y)^{n}
$$

where

$$
\begin{aligned}
\frac{a_{10}}{a_{0} k_{1}^{2}} & =n, \\
a_{0} & =a, \\
a_{3} & =\frac{k_{1}}{k_{2}} b, \\
A_{0} & =a X_{0}+b Y_{0}, \\
X+X_{0} & =x, \\
Y+Y_{0} & =y .
\end{aligned}
$$

Using equation (36) and similarity requirement (viii) of equation (26), one may obtain

$$
U_{e}(x, y)=U_{0}(a x+b y)^{m}
$$

where $m=a_{7} / a_{0}$. From other requirements, $\gamma^{2}(x, y)$ and $\Delta T$ $(x, y)$ are found to be in this situation

$$
\begin{aligned}
\gamma^{2}(x, y) & =(a x+b y)^{n+1-m}, \\
\Delta T(x, y) & =(\Delta T)_{0}(a x+b y)^{2 m-n-1},
\end{aligned}
$$

here, $U_{0}$ and $(\Delta T)_{0}$ are integrating constants that represent velocity and temperature difference, respectively.

Substituting equations (37), (38), (39), and (40) in the similarity requirements, the constants in terms of $a$ and $b$ associated with $m, n, k_{1}$ are

$$
\begin{aligned}
& a_{0}=a, \\
& a_{1}=(m+n) a, \\
& a_{2}=-2 n a, \\
& a_{3}=\frac{k_{1}}{k_{2}} a, \\
& a_{4}=(m+n) \frac{k_{1}}{k_{2}} b, \\
& a_{5}=-2 n a, \\
& a_{7}=m a, \\
& a_{8}=m \frac{k_{1}}{k_{2}} b, \\
& a_{9}=n \frac{k_{1}}{k_{2}} b, \\
& a_{10}=k_{1}^{2} n a, \\
& a_{12}=m \frac{k_{1}}{k_{2}} b, \\
& a_{13}=m a, \\
& a_{14}=n a, \\
& a_{15}=\frac{n a}{k_{1}^{2}}, \\
& a_{18}=(2 m-n-1) a, \\
& a_{1}
\end{aligned} .
$$

Hence, transform equations (23)-(25) become

$$
\begin{aligned}
& v \bar{F}_{\overline{\phi \phi \phi}}+\frac{1}{2}(3 n+m+1) a \bar{F}_{\overline{\phi \phi}}+\frac{1}{2}(3 n+m+1) \frac{k_{1}}{k_{2}} b \bar{S} \bar{F}_{\overline{\phi \phi}} \\
& +m a\left(1-\bar{F}_{\bar{\phi}}^{2}\right)+(m+n) \frac{k_{1}}{k_{2}} b\left(1-\bar{F}_{\bar{\phi}} \bar{S}_{\bar{\phi}}\right) \\
& \quad-a_{6} \bar{F}_{\overline{\phi \phi}}-k_{1}^{2} n a\left(1-\bar{S}_{\bar{\phi}}^{2}\right)+a_{11} \bar{\theta}=0,
\end{aligned}
$$

$$
\begin{aligned}
& v \bar{S}_{\overline{\phi \phi \phi}}+\frac{a}{2}(3 n+m+1) \bar{F} \bar{S}_{\overline{\phi \phi}}+\frac{b k_{1}}{2 k_{2}}(3 n+m+1) \bar{S} \bar{S}_{\overline{\phi \phi}} \\
& +\frac{b m k_{1}}{2 k_{2}}\left(1-\bar{S}_{\bar{\phi}}^{2}\right)+a(m+n)\left(1-\bar{F}_{\bar{\phi}} \bar{S}_{\bar{\phi}}\right)-a_{6} \bar{S}_{\overline{\phi \phi}} \\
& \quad-\frac{m b}{k_{1} k_{2}}\left(1-\bar{F}_{\bar{\phi}}^{2}\right)+a_{16} \bar{\theta}=0, \\
& v P_{r}^{-1} \bar{\theta}_{\overline{\phi \phi}}+\frac{1}{2}(3 n+m+1)\left[a \bar{F}+\frac{k_{1}}{k_{2}} b \bar{S}\right] \bar{\theta}_{\bar{\phi}}-a_{6} \bar{\theta}_{\bar{\phi}} \\
& \quad-(2 m-n-1)\left[a \bar{F}_{\bar{\phi}}+\frac{k_{1}}{k_{2}} b \bar{S}_{\bar{\phi}}\right] \bar{\theta}+a_{19} \bar{\theta}=0 .
\end{aligned}
$$

In order to simplify the above type of equations, we substitute $\bar{F}=\alpha f, \bar{S}=\alpha g, \bar{\theta}=\vartheta$, and $\bar{\phi}=\alpha \phi$. Choosing $(a(3 n+$ 
TABLE 1: Similarity requirements in curvilinear coordinates.

\begin{tabular}{lcccc}
\hline$h_{1}$ & $h_{2}$ & $U_{e}$ & $V_{e}$ & $\Delta T$ \\
\hline$(a x+b y)^{n}$ & $k_{2} h_{1}$ & $(a x+b y)^{m}$ & $k_{1} U_{e}$ & $(a x+b y)^{2 m-n-1}$ \\
$(a x+b y)^{n}$ & $k_{2} h_{1}$ & $\exp \{n(a x+b y)\}$ & $k_{1} U_{e}$ & {$\left[\frac{\exp (a x+b y)}{(a x+b y)}\right]^{n}$} \\
$\exp (a x+b y)$ & $k_{2} h_{1}$ & $\exp \{(a x+b y)\}$ & $k_{1} U_{e}$ & $\exp \{(a x+b y)\}$ \\
1 & $x^{n}$ & $x^{m}$ & $k_{1} U_{e}$ & $x^{2 m-1}$ \\
1 & $\exp (n x)$ & $\exp (m x)$ & $k_{1} U_{e}$ & $\exp (2 m x)$ \\
$y^{n}$ & 1 & $y^{m}$ & $k_{1} U_{e}$ & $y^{2 m-1}$ \\
$\exp (n y)$ & 1 & $\exp (m y)$ & $k_{1} U_{e}$ & $\exp (2 m y)$ \\
\hline
\end{tabular}

$\left.m+1) \alpha^{2}\right) / 2 v=1, \quad k_{1} b / k_{2} a=c, 2 a_{6} /(\alpha(3 n+m+1) a)=-P_{w}$, and $\beta=2 a_{19} /((3 n+m+1) a)$, the final form of the similarity solution stands as

$$
\begin{gathered}
f_{\phi \phi \phi}+(f+c g) f_{\phi \phi}+\left(\frac{2 m}{3 n+m+1}\right)\left(1-f_{\phi}^{2}\right) \\
+2 c\left(\frac{m+n}{3 n+m+1}\right)\left(1-f_{\phi} g_{\phi}\right)+P_{w} f_{\phi \phi} \\
-\left(\frac{2 n k_{1}^{2}}{3 n+m+1}\right)\left(1-g_{\phi}^{2}\right)+\frac{U_{F}^{2}}{U_{e}^{2}} \vartheta=0, \\
g_{\phi \phi \phi}+(f+c g) g_{\phi \phi}+\left(\frac{2 m c}{3 n+m+1}\right)\left(1-g_{\phi}^{2}\right) \\
+2\left(\frac{m+n}{3 n+m+1}\right)\left(1-f_{\phi} g_{\phi}\right)+P_{w} g_{\phi \phi} \\
-\frac{2 c}{k_{1}^{2}}\left(\frac{n}{3 n+m+1}\right)\left(1-f_{\phi}^{2}\right)+\frac{V_{F}^{2}}{V_{e}^{2}} \vartheta=0, \\
P_{r}^{-1} \vartheta_{\phi \phi}+(f+c g) \vartheta_{\phi}+P_{w} \vartheta_{\phi}-2\left(\frac{2 m-n-1}{3 n+m+1}\right) \\
\cdot\left(f_{\phi}+c g_{\phi}\right) \vartheta+\beta \vartheta=0 .
\end{gathered}
$$

$\alpha$ is introduced here in order to reduce the types of equations (42), (43), and (44) in a simplified form as equations (45), (46), and (47).

$U_{F}^{2}=-g_{x} \beta_{T} \Delta T h_{1} \cdot$ characteristic length,

$V_{F}^{2}=-g_{y} \beta_{T} \Delta T h_{2} \cdot$ characteristic length,

$$
\text { characteristic length }=\frac{a x+b y}{a(3 n+m+1)} .
$$

The boundary conditions are

$$
\begin{aligned}
& f(0)+c g(0)=P_{w}, \quad f_{\varphi}(0)=0, \quad f_{\varphi}(\infty)=1, \\
& g(0)=g_{0}, \quad g_{\phi}(0)=0, \quad g_{\phi}(\infty)=1, \\
& \vartheta(0)=1, \quad \vartheta(\infty)=0 .
\end{aligned}
$$

In this case, velocity component $w$ perpendicular to the surface and the similarity variable $\phi$ are found to be

$$
\begin{aligned}
w= & \left(\frac{v a}{2}\right)^{1 / 2}\left(\frac{3 n+m+1}{2}\right)^{1 / 2}(a x+b y)^{(m-n-1) / 2} \\
& \cdot\left[f+c s-\phi\left(\frac{n+1-m}{3 n+m+1}\right)\left(f_{\phi}+c s_{\phi}\right)\right], \\
\phi= & z\left[\frac{a(3 n+m+1)}{2 v}\right]^{1 / 2}(a x+b y)^{(m-n-1) / 2} .
\end{aligned}
$$

For a real situation, the condition of irrotationality in the main stream $\left(h_{2} V_{e}\right)_{x}-\left(h_{1} U_{e}\right)_{y}=0$, yields $m=-n$ or $c=k_{1}^{2}$. If $m \neq-n$, equations (45), (46), and (47) with boundary condition (49) are

$$
\begin{gathered}
f_{\phi \phi \phi}+(f+c g) f_{\phi \phi}+\left(\frac{2 m}{3 n+m+1}\right)\left(1-f_{\phi}^{2}\right) \\
+2 c\left(\frac{m+n}{3 n+m+1}\right)\left(1-f_{\phi} g_{\phi}\right)+P_{w} f_{\phi \phi} \\
-\left(\frac{2 n c}{3 n+m+1}\right)\left(1-g_{\phi}^{2}\right)+\frac{U_{F}^{2}}{U_{e}^{2}} \vartheta=0, \\
g_{\phi \phi \phi}+(f+c g) g_{\phi \phi}+\left(\frac{2 m c}{3 n+m+1}\right)\left(1-g_{\phi}^{2}\right) \\
+2\left(\frac{m+n}{3 n+m+1}\right)\left(1-f_{\phi} g_{\phi}\right)+P_{w} g_{\phi \phi} \\
-2\left(\frac{n}{3 n+m+1}\right)\left(1-f_{\phi}^{2}\right)+\frac{V_{F}^{2}}{V_{e}^{2}} \vartheta=0, \\
P_{r}^{-1} \vartheta_{\phi \phi}+(f+c g) \vartheta_{\phi}+P_{w} \vartheta_{\phi}-2\left(\frac{2 m-n-1}{3 n+m+1}\right) \\
\cdot\left(f_{\phi}+c g_{\phi}\right) \vartheta+\beta \vartheta=0 .
\end{gathered}
$$

Equations (37), (38), (39), and (40) are the requirement for the existence of one of the possible similarity solutions. Equations (51), (52), and (53) with the boundary conditions (49) are the similarity solutions in which the mainstream has the velocity components $\left(U_{e}, V_{e}, 0\right)$ satisfying the condition of irrotationality. In similar way, other possible cases in 
curvilinear coordinates like equations (37), (38), (39), and (40) are given a tabular form.

\section{Conclusions}

General discussion for similarity requirements, detail similarity analyses have been done presented in Table 1 to reduce the governing partial differential equations into the set of ordinary differential equations for an incompressible fluid flow over the curvilinear surfaces. Detail discussion of one case has been presented in this paper, and different possible cases are exhibited in a tabular form with the inclusion of $\Delta T, h_{1}, h_{2}, U_{e}$, and $V_{e}$ variations for onward flow study that are helpful to the future researchers for the flow over the orthogonal curvilinear surfaces. If we put $c=0, n=0$, and $P_{w}=0$, the above equations (51)-(52) become wellknown Falkner-Skan equation in the absence of $U_{F}=0$ and $\beta=0$, i.e., buoyancy and heat absorption effects. If $c=0$, $n=0, k_{1}=0, U_{F}=0, \beta=0$, and $P_{w}=0$, the equations coincide with those dealt with by Sparrow et al. [31].

\section{Data Availability}

The data used to support the findings of this study are available from the corresponding author upon request.

\section{Conflicts of Interest}

The authors declare that they have no conflicts of interest.

\section{References}

[1] L. Howarth, "XXV. The boundary layer in three dimensional flow. -Part I. Derivation of the equations for flow along a general curved surface," The London, Edinburgh, and Dublin Philosophical Magazine and Journal of Science, vol. 42, no. 326, pp. 239-243, 1951.

[2] A. Davey and D. Schofield, "Three-dimensional flow near a two-dimensional stagnation point," Journal of Fluid Mechanics, vol. 28, no. 1, pp. 149-151, 1967.

[3] A. G. Hansen, Similarity Analyses of Boundary Layer Problems in Engineering, Prentice-Hall, Inc, Englewood, Cliffs, N. J., 1964.

[4] A. G. Hansen, "Similarity solutions of a class of laminar threedimensional boundary layer equations of power law fluids," International Journal of Non-Linear Mechanics, vol. 2, no. 4, pp. 373-385, 1967.

[5] K. C. Wang, "Boundary layer over a blunt body at high incidence with an open-type of separation," Proceedings of the Royal Society A: Mathematical, Physical and Engineering Sciences, vol. 340, no. 1620, pp. 33-55, 1974.

[6] P. Cheng, "Combined forced and free convection flow about inclined surfaces in porous media," International Journal of Heat and Mass Transfer, vol. 20, no. 8, pp. 807-814, 1977.

[7] L. S. Langston, M. L. Nice, and R. M. Hooper, "Three-dimensional flow within a turbine cascade passage," Journal of Engineering for Power, vol. 99, no. 1, pp. 21-28, 1977.

[8] S. M. Raju, Q. X. Liu, and K. C. Law, "A formulation of combined forced and free convection past horizontal and vertical surfaces," International Journal of Heat and Mass Transfer, vol. 27, no. 12, pp. 2215-2224, 1984.
[9] J. H. Merkin and T. Mahmood, "On the free convection boundary layer on a vertical plate with prescribed surface heat flux," Journal of Engineering Mathematics, vol. 24, no. 2, pp. 95-107, 1990.

[10] M. S. Chong, A. E. Perry, and B. J. Cantwell, "A general classification of three-dimensional flow fields," Physics of Fluids, vol. 2, no. 5, pp. 765-777, 1990.

[11] H. T. Chen and S. M. Chang, "Numerical simulation for conjugate problem of natural convection on both sides of a vertical wall," International Journal of Heat and Mass Transfer, vol. 39, no. 2, pp. 383-390, 1996.

[12] M. Zakerullah and K. A. Maleque, "Laminar combined convective flow about a vertical inclined surface," Journal of Bangladesh Academy of Sciences, vol. 20, no. 2, pp. 99-110, 1996.

[13] M. Zakerullah and K. A. Maleque, "Similarity solutions for mixed convective flow about a rectangular vertical inclined plane surface," Journal of Bangladesh Mathematical Society, vol. 18, pp. 9-29, 1998.

[14] M. A. Sattar and K. A. Maleque, "Numerical solution of MHD free convection and mass transfer flow with Hall current, viscous dissipation and Joule heating," Journal of Energy, Heat and Mass Transfer, vol. 22, pp. 237-244, 2000.

[15] K. A. Maleque and M. A. Sattar, "Transient convective flow due to a rotating disc with magnetic field and heat absorption effects," Journal of Energy, Heat and Mass Transfer, vol. 25, pp. 279-291, 2003.

[16] K. A. Maleque and M. A. Sattar, "MHD convective flow due to a rotating disk with Hall effect," Journal of Energy, Heat and Mass Transfer, vol. 27, pp. 211-228, 2005.

[17] K. A. Maleque and M. A. Sattar, "Steady laminar convective flow with variable properties due to a porous rotating disk," ASME Journal of Heat Transfer, Technical Brief, vol. 127, no. 12, pp. 1406-1409, 2005.

[18] K. A. Maleque and M. A. Sattar, "The effects of variable properties and Hall current on steady MHD compressible laminar convective fluid flow due to a porous rotating disc," International Journal of Heat and Mass Transfer, vol. 48, pp. 49634972, 2005.

[19] K. A. Maleque, "Magnetohydrodynamic convective heat and mass transfer flow due to a rotating disk with thermal diffusion effect," Journal of heat transfer, vol. 131, no. 8, article 082001, 2009.

[20] L. U. Schrader, L. Brandt, and D. S. Henningson, "Receptivity mechanisms in three-dimensional boundary-layer flows," Journal of Fluid Mechanics, vol. 618, pp. 209-241, 2009.

[21] K. A. Maleque, "Effects of combined temperature- and depthdependent viscosity and Hall current on an unsteady MHD laminar convective flow due to a rotating disk," Chemical Engineering Communications, vol. 197, no. 4, pp. 506-521, 2010.

[22] K. A. Maleque, "Dufour and Soret effects on unsteady MHD convective heat and mass transfer flow due to a rotating disk," The Latin American Applied Research Journal, vol. 40, no. 2, pp. 105-111, 2010.

[23] S. Nadeem, R. U. Haq, N. S. Akbar, and Z. H. Khan, "MHD three-dimensional Casson fluid flow past a porous linearly stretching sheet," Alexandria Engineering Journal, vol. 52, no. 4, pp. 577-582, 2013.

[24] S. Nadeem, R. U. Haq, and N. S. Akbar, "MHD threedimensional boundary layer flow of Casson nanofluid past a linearly stretching sheet with convective boundary condition," 
IEEE Transactions on Nanotechnology, vol. 13, no. 1, pp. 109115, 2014.

[25] M. A. Mohd Noor and N. Roslinda, "Numerical solutions of three-dimensional boundary layer flow and heat transfer past a permeable shrinking surface in a $\mathrm{Cu}$-water nanofluid," $A I P$ Conference Proceedings, vol. 1602, p. 192, 2014.

[26] T. Hayat, M. Taseer, S. A. Shehzad, and A. Alsaedi, "A mathematical study for three-dimensional boundary layer flow of Jeffrey nanofluid," Zeitschrift für Naturforschung A, vol. 70, no. 4, pp. 225-233, 2015.

[27] K. A. Maleque, "Unsteady MHD non-Newtonian Casson fluid flow due to a porous rotating disk with uniform electric field," Fluid Mechanics: Open Access, vol. 3, no. 1, p. 123, 2016.

[28] A. Maleque Kh, "Effect of hall current on MHD nonNewtonian unsteady Casson fluid porous rotating disk flow with a uniform electric field," AIUB Journal of Science and Engineering (AJSE), vol. 16, no. 2, pp. 51-60, 2017.

[29] T. Hayat, M. Z. Kayani, A. Alsaedi, M. I. Khan, and I. Ahmad, "Mixed convective three-dimensional flow of Williamson nanofluid subject to chemical reaction," International Journal of Heat and Mass Transfer, vol. 127, pp. 422-429, 2018.

[30] S. Rashid, M. I. Khan, T. Hayat, M. Ayub, and A. Alsaedi, "Numerical treatment for rotating Maxwell nanomaterial flow with Arrhenius energy," Applied Nanoscience, 2019.

[31] E. M. Sparrow, R. Eichhorn, and L. J. Gregg, "Combined forced and free convection in a boundary layer flow," Physics of Fluids, vol. 2, no. 3, p. 319, 1959. 